\title{
Overview of the Health Informatics Research Field: A Bibliometric Approach
}

\author{
Hai-Ning Liang \\ National ICT Australia (NICTA), \\ Queensland Research Lab, Brisbane, Australia \\ hai-ning.liang@nicta.com.au
}

\begin{abstract}
Health informatics is a relatively new research area. Over the last decade or so, research in health information has been growing at a very rapid rate, as evidenced by the large number of publications. While this growth has been beneficial to the field, it has also made understanding the scope of the field more difficult. Consequently, it is difficult to answer questions such as how the research field has evolved over time, what the landmark publications are, what impact these publications have had, and who are the most prolific and high impact researchers. The purpose of this paper is to provide an overview of the research field of health informatics and, in doing so, attempt to answer some of the questions just mentioned. To this end, we make use of two bibliometric tools: HistCite and CiteSpace II. Because these tools offer complementary bibliometric methods, their use together provides results that are more robust. In this paper, we report some general findings of our bibliometric analysis using these two tools.
\end{abstract}

Keywords: Health informatics; medical informatics; e-health; bibliometrics; literature review; research field; citation and co-citation analysis.

\section{Introduction and Background}

The term 'health informatics' came into existence around 1973 [1]. For the last decade or so health informatics research has been growing at a rapid rate, partly fuelled by advances in information technologies and partly by an urgent need to improve quality of care and patient safety [2,3]. Although there are several definitions for health informatics, Coiera [3] suggests that it is "the study of information and communication systems in healthcare" (p.xxii). Health informatics is thought to be situated at the crossroad of information science, computer science, medicine, and healthcare, with a wide range of application areas including nursing, clinical care, public health, and biomedicine [3,4].

Because of its multidisciplinary foundations and diverse application areas, health informatics has captured the interest of researchers from numerous areas. Engagement from these researchers has been beneficial to the field as it has led to the development of a rich research literature appearing in many journals. Consequently, because the corpus of literature has become so complex, it is difficult for newcomers to the area to grasp its foundations and main themes, and even for those versed in the area to stay abreast of new developments. 
As research in health informatics continues to grow, it may be useful to have a clearer understanding of its major themes. Perhaps only then will it be possible to answer questions such as how the field has evolved over time, what its landmark publications are, what impact these landmark publications have had, and who have been the most prolific and high impact researchers.

The purpose of this paper is to provide an overview of research field of health informatics. We use bibliometric methods [5-8], which are formal, quantifiable techniques for analysing research activities and output, such as publications and patents. Bibliometric methods are becoming more popular; for example, the UK government is looking into the possibility of using such methods to assess the quality of research publications of UK universities [see 9]. These methods require an aggregation of publications, and when bibliometric methods are applied to the aggregation, it is possible to identify statistical and structural properties of citation, co-citation, or co-author networks and patterns.

There are currently several computer-based biblimetric tools such as Bibexel [10], CiteSpace II [11], HistCite [12], and Sitkis [13], each of which operationalizes certain bibliometric methods. These tools allow for a more automated analysis of research output in order to determine the impact of a field, of a set of researchers, or of a specific publication.

We selected two bibliometric tools, CiteSpace II [11, 14] and HistCite [15, 16], to assist in the analysis of the research field of health informatics, and in doing so, we attempt to find answers to the questions noted above.

\section{Method}

\subsection{CiteSpace II and HistCite}

An important step of this research was to survey the available bibliometric tools. Four potential tools were identified: Bibexel [10], CiteSpace II [11], HistCite [12], and Sitkis [13] ${ }^{1}$. Broadly speaking, these tools can be classified as visualization-based or text-based. Visualization-based tools emphasize the use of graphical means in the exploration and analysis of patterns in research productivity. These tools provide their output as visual representations through which patterns and trends can be detected by visual inspection. One attractive feature of visualization-based tools is that their visual representations are often interactive and so are directly responsive to analysts' needs [17]. Text-based tools, on the other hand, give more primacy to the use of tables and charts as outputs for inspection and analysis.

We selected one tool from each group: CiteSpace II and HistCite. CiteSpace II is visualization-based whereas HistCite is text-based. Another criterion for the choice was that each offers features not available in the other. CiteSpace II bases its analysis on co-citation counts. HistCite, on the other hand, bases its analysis on citation counts. Because the tools are complementary, using them together may add to the validity of our analysis.

\footnotetext{
${ }^{1}$ HistCite is the only commercial tool.
} 


\subsection{Data Collection}

We conducted a literature search on the ISI Web of Knowledge, Web of Science databases [18], which include Science Citation Index Expanded, Social Sciences Citation Index, Arts \& Humanities Citation Index, and Conference Proceedings Citation Index - both Science, and Social Science \& Humanities. We intentionally made our search as broad as possible using the terms "medical informatics", "health informatics", "eHealth", and "e-Health". Overall, our search returned 3708 results" The search results were entered into both CiteSpace II and HistCite for analysis.

\section{Results}

\subsection{Descriptive Results}

Figure 1 shows the growth of research output since 1973 within the scope of our query, and Table 1 displays the output per year since 1973. Several important observations can be made. First, the first publications appeared in the early seventies. The first World Congress on Medical Informatics took place during this time [19]. Second, growth in the number of publications in the seventies, eighties, and early nineties was slow. Third, there were five key years in which publications jumped in numbers: 1992-1993, 1996-1997, 2000-2001, 2003-2004, and 2006-2007 (highlighted in Table 1). In short, if this trend continues, we will expect to see the number of publications in the next few years to be around 400-500, if not higher.

The search collection contains 8472 authors and co-authors. Table 2 shows the most productive authors - those who have published at least fifteen papers. The most productive author is R. Haux with 73 papers, having more than twice the number of publications than the next author, E. Ammenwerth, who is closely followed by $\mathrm{P}$. Knaup, and A. Hasman. As discussed further down, the work of these researchers, especially of R. Haux, seems to have had great impact in the field, particularly in laying its initial foundations.

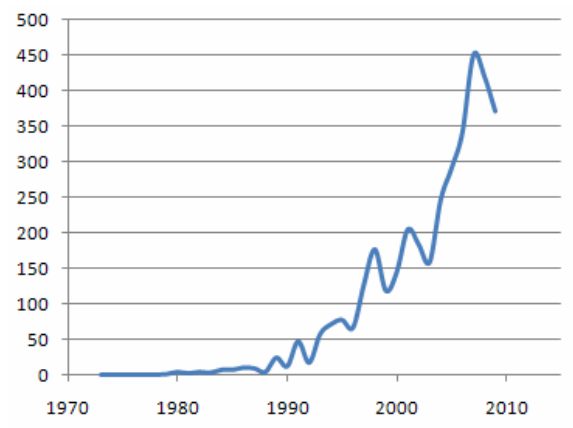

Table 1. Yearly output of publications (adapted from HistCite)

\begin{tabular}{|c|c|c|c|c|c|}
\hline 1973 & 1 & 1986 & 11 & 1998 & 177 \\
\hline 1974 & 1 & 1987 & 10 & 1999 & 119 \\
\hline 1976 & 1 & 1988 & 5 & 2000 & 145 \\
\hline 1977 & 1 & 1989 & 25 & $\mathbf{2 0 0 1}$ & $\mathbf{2 0 5}$ \\
\hline 1978 & 1 & 1990 & 13 & 2002 & 184 \\
\hline 1979 & 2 & 1991 & 48 & 2003 & 159 \\
\hline 1980 & 5 & 1992 & 18 & $\mathbf{2 0 0 4}$ & $\mathbf{2 4 6}$ \\
\hline 1981 & 3 & $\mathbf{1 9 9 3}$ & $\mathbf{5 8}$ & 2005 & 291 \\
\hline 1982 & 5 & 1994 & 72 & 2006 & 342 \\
\hline 1983 & 4 & 1995 & 78 & $\mathbf{2 0 0 7}$ & $\mathbf{4 5 0}$ \\
\hline 1984 & 8 & 1996 & 67 & 2008 & 420 \\
\hline 1985 & 8 & $\mathbf{1 9 9 7}$ & $\mathbf{1 2 8}$ & 2009 & 371 \\
\hline
\end{tabular}

Fig. 1. Growth of publications in the collection

\footnotetext{
${ }^{2}$ Search was in January, 2010.
} 
Table 3 presents the list of top research institutions based on output. The Harvard School of Medicine is ranked first with 42 publications. The National Library of Medicine is next with 30 publications, followed by the School of Health Information Science at the University of Victoria with 28 publications. The papers in the collection have come predominantly from research institutions in the US (9 out of 14), followed by institutions in Germany (3 out of 14).

Table 2. Authors with at least fifteen papers in the collection (adapted from HistCite)

\begin{tabular}{ccl}
\hline Rank & \# Papers & \multicolumn{1}{c}{ Authors } \\
\hline 1 & 73 & Haux, R. \\
2 & 34 & Ammenwerth, E. \\
3 & 30 & Knaup, P. \\
4 & 28 & Hasman, A. \\
5 & 21 & Wyatt, J.C. \\
6 & 20 & Moehr, J.R. \\
7 & 20 & Shortliffe, E.H. \\
8 & 19 & Blobel, B. \\
9 & 19 & Maojo, V. \\
10 & 18 & Cimino, J.J. \\
11 & 18 & Timpka, T. \\
12 & 17 & Ball, M.J. \\
13 & 17 & Eysenbach, G. \\
14 & 17 & Gardner, R.M. \\
15 & 17 & Patel, V.L. \\
16 & 16 & Rigby, M. \\
17 & 16 & Sullivan, F. \\
18 & 15 & Leven, F.J. \\
19 & 15 & Martin-Sanchez, F. \\
\hline
\end{tabular}

Table 3. List of top research institutions (adapted from HistCite)

\begin{tabular}{llll}
\hline Rank & \# Papers & Institutions/Subdivision & Country \\
\hline 1 & 42 & Harvard University, School of Medicine & USA \\
2 & 30 & National Library of Medicine & USA \\
3 & 28 & University of Victoria, School of Health Information Science & Canada \\
4 & 25 & University of Heidelberg, Department of Medical Information & Germany \\
5 & 24 & Tech Univ Carolo Wilhelmina Braunschweig, Inst Med Informat & Germany \\
6 & 20 & Univ Amsterdam, Acad Med Ctr & Holland \\
7 & 19 & Yale Univ, Sch Med & USA \\
8 & 19 & Rutgers State Univ, Dept Comp Sci & USA \\
9 & 19 & Univ Heidelberg, Inst Med Biometry \& Informat & Germany \\
10 & 19 & Vanderbilt Univ, Med Ctr & USA \\
11 & 17 & Indiana Univ, Sch Med & USA \\
12 & 17 & Stanford Univ, Sch Med & USA \\
13 & 17 & Univ Utah, Sch Med & USA \\
14 & 15 & Univ Texas, Hlth Sci Ctr & USA \\
\hline
\end{tabular}

Finally, Table 4 shows the most important journals according to how many publications described by our search terms were found in the journal. The three top journals are Methods of Information in Medicine, International Journal of Medical Informatics, and Journal of the American Medical Informatics Association, with 295, 271 , and 233 publications respectively. 
Table 4. Top journals based on publication output (adapted from HistCite)

\begin{tabular}{lllc}
\hline Rank & \# Papers & Journals & Impact Factor $^{3}$ \\
\hline 1 & 295 & Methods of Information in Medicine & 1.1 \\
2 & 271 & International Journal of Medical Informatics & 2.8 \\
3 & 233 & Journal of the American Medical Informatics Association & 3.4 \\
4 & 79 & Telemedicine Journal and E-Health & 1.4 \\
5 & 54 & Journal of Medical Internet Research & 3.6 \\
6 & 52 & Journal of Telemedicine and Telecare & 0.9 \\
7 & 42 & Connecting Medical Informatics and Bio-Informatics & NA \\
8 & 42 & Journal of Biomedical Informatics & 1.9 \\
9 & 35 & Computer Methods and Programs in Biomedicine & 1.2 \\
10 & 35 & M D Computing & NA \\
11 & 30 & British Medical Journal & 12.9 \\
\hline
\end{tabular}

\subsection{Results from Citation Analysis}

\subsubsection{Citation Analysis}

As noted earlier, HistCite uses citation counts for its bibliometric calculations. It relies on two main measures: (1) Local Citation Score (LCS) which indicates the count of citations to a paper within the collection; and, (2) Global Citation Score (GCS) which represents the total number of citations to a paper in the Web of Science, regardless of the whether citing papers belong to the collection or not. Using GCS, LCS, and other measures, HistCite calculates the impact of a particular publication or author [12]. The output of HistCite can be "used to help the searcher quickly identify the most significant work on a topic and trace its year-by-year historical development" [12, p.119].

Table 5. Publication ranking based on Local Citation Score (Adapted from HistCite)

\begin{tabular}{lcccc}
\hline \multicolumn{1}{c}{ Articles } & LCS & Rank (LCS) & GCL & Rank (GCL) \\
\hline Greenes \& Shortliffe [20] & 83 & 1 & 157 & 5 \\
Haux \& Knaup [22] & 41 & 2 & 50 & 43 \\
Eysenbach [23] & 32 & 3 & 126 & 6 \\
Kaplan [24] & 32 & 4 & 88 & 16 \\
Gustafson et al. [25] & 30 & 5 & 161 & 4 \\
Ammenwerth et al. [26] & 30 & 6 & 48 & 47 \\
vanBemmel [27] & 27 & 7 & 38 & 74 \\
Kaplan [28] & 24 & 8 & 68 & 24 \\
Martin-Sanchez [29] & 24 & 9 & 40 & 66 \\
Sittig [30] & 22 & 10 & 38 & 72 \\
Haux [31] & 22 & 11 & 25 & 146 \\
Lorenzi et al. [32] & 22 & 12 & 69 & 23 \\
Kulikowski [33] & 22 & 13 & 24 & 160 \\
Collen [21] & 21 & 14 & 26 & $>200$ \\
Stead et al. [34] & 21 & 15 & 51 & 41 \\
Heathfield \& Wyatt [35] & 21 & 16 & 24 & 156 \\
\hline
\end{tabular}

Table 5 shows the ranking of articles based on LCS and GCS measures. It seems that the LCS measure provides a more accurate representation of the relevance of

\footnotetext{
${ }^{3}$ Numbers were obtained from each journal's website (visited on February 2010).
} 
publications to the search terms than the GCS measure. When using the GCS measure as the main criterion, in contrast, some high-ranking articles are not so relevant to health informatics. The publications from Table 5 include articles that seem to have provided the foundations and created the initial momentum for health informatics as a research discipline.

One of the most useful features of HistCite is its ability to produce a historiograph of the most-cited articles in the collection (see Figure 2). The nodes represent publications and the links between them depict citations. An arrow pointing from one node back to another indicates that the article at the origin of the arrow cites the article at the end of the arrow. For example, article 99 [20] cites article 50 [21]. The size of the node at the end of the arrow is proportional to how often it is cited.

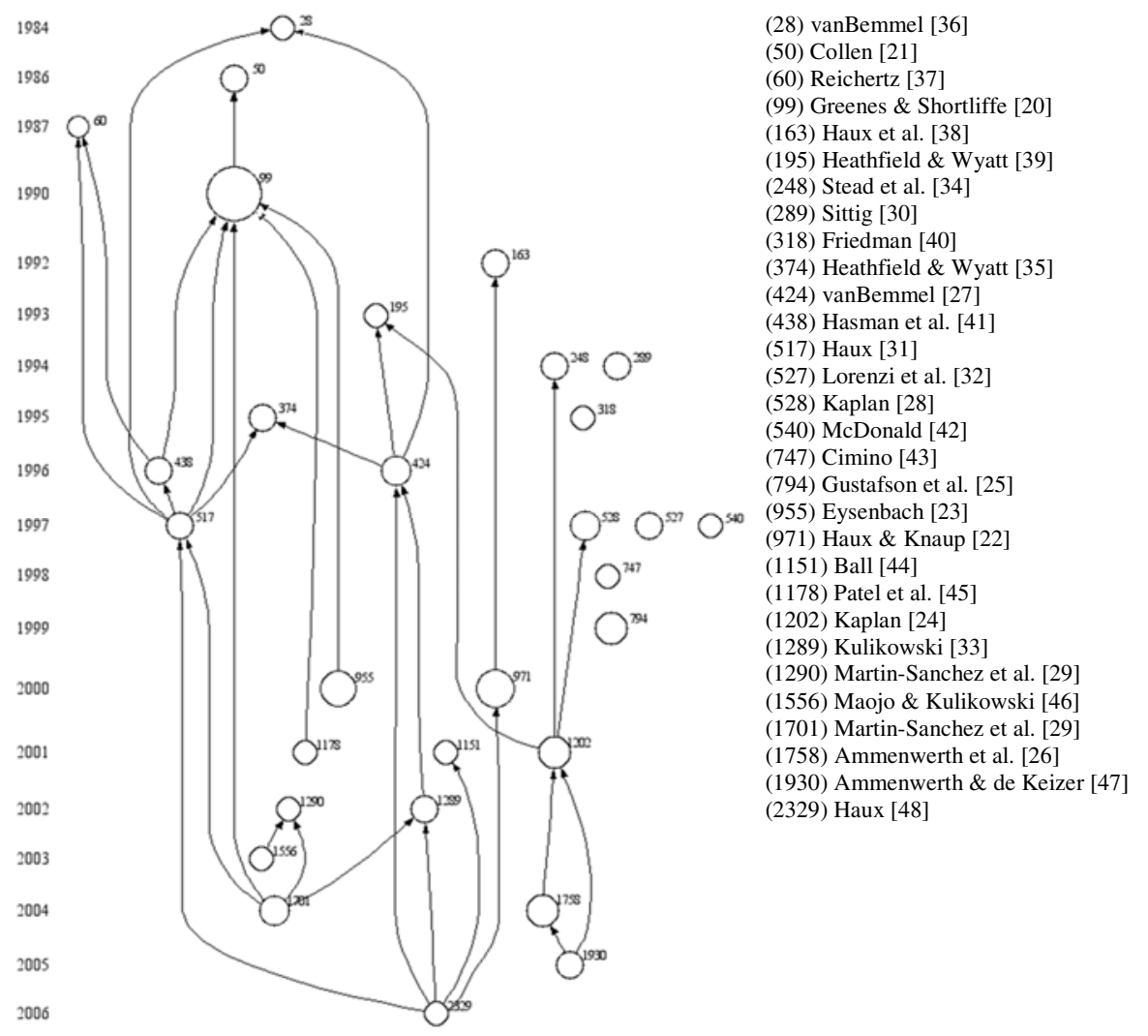

Fig. 2. Historiograph of the 30 most cited publications (based on LCS) in the collection (adapted from HistCite)

The threshold number is user-defined; and the user can choose to use either LCS or GLS as the main criterion. Figure 2 shows the 30 most-cited articles in terms of LCS-i.e., citation made from the articles within the collection. Greenes and Shortliffe's article [20] (node 99; published in 1990) is the most dominant node in the collection, followed by articles from Eysenbach [23] (node 955; published in 2000) 
and Haux and Knaup [22] (node 971; also published in 2000). These three articles are the top three items of Table 5. What Table 5 does not show, but which Figure 2 does, is how the most cited articles are related. A closer look at these articles indicates that they are conceptual in nature, and attempt to provide the foundations for health informatics as a field. For example, Greenes and Shortliffe [20] make a strong case for health informatics to be an academic discipline by describing its scope, some basic research problems, and potential career paths for graduates. In general, most of the articles in Figure 2 are conceptual and endeavor to define the aims and tasks of medical informatics [31]; to describe its origins [21]; to provide a systematic view [31]; to delineate its grand challenges [20]; point out its structure [36]; to provide some direction for future research $[26,48]$.

In term of how the articles are related, according to Figure 2, one of the key articles is by vanBemmel [36], published in 1984, which has been cited by Haux [31] and self-cited by another of vanBemmel's articles [27]. The next two important articles are from Collen [21] and [37]. Greenes and Shortliffe's [20] seminal article comes next, citing Collen's 1986 article and it is cited by several important articles in the collection [23, 29, 31, 41, 45]. The influence of Greenes and Shortliffe's article trickles down to a recent article in the collection from Haux [48], where the past, present, and future of health information systems is described. Further relationships can be discerned from Figure 2. A deeper analysis of the content of the articles is needed to ascertain the degree of the influence of the different articles. This level of analysis is beyond the scope of this paper.

\subsubsection{Co-citation Analysis}

The above analysis is based mainly on the output of HistCite, which relies primarily on citation counts. To complement this analysis, another bibliometric tool, CiteSpace II [11], is used to analyze the same collection of search results. Unlike HistCite, CiteSpace II relies on both citation and co-citation counts. In addition, with the exception of the historiograph, HistCite provides outputs that are text-based; whereas CiteSpace II's main outputs are visualizations, which are intended to support the detection of trends and patterns in a body of literature. CiteSpace II's visualizations are interactive, and include illustrations of co-citation networks. By specifying different threshold levels, e.g., for citation and co-citation counts, it may be possible to (1) determine influential publications for a particular period; and (2) observe trends and patterns within the body of literature.

In CiteSpace II, the entire time interval is divided into equal segments (e.g., 1-year, 2-year, 3-year, and so on). For each segment, CiteSpace II creates a co-citation network, and integrates all the networks into one single synthesized visualization network. The publications in the collection extend from the early 1970s to the present. Because of the large number of publications, the resulting network of all years included as one continuous interval is very complex. Therefore, we divided the analysis into three intervals: 1970-2000, 2001-2005, and 2006-2009.

Figure 3 shows the resulting network of co-citations for the first interval (19702000). Nodes in the network depict publications. The nodes are represented as concentric rings, where the thickness of a ring is proportional to the number of citations in a given time slice. The size of the outermost ring and the size of the font are proportional to the centrality of the publication. The links depict co-citations, and 
their color represent the year of the first co-citation of the publication. As can be observed from Figure 3, the main articles that are also the most influential articles are: Greenes [20], Heathfield [35], vanBemmel [27], and Lorenzi [32]. The dominant color of the links is orange and corresponds to co-citations which were first made during the 1990-2000 timeframe. The other salient colors, yellow and green, depict co-citations first made prior to 1990.

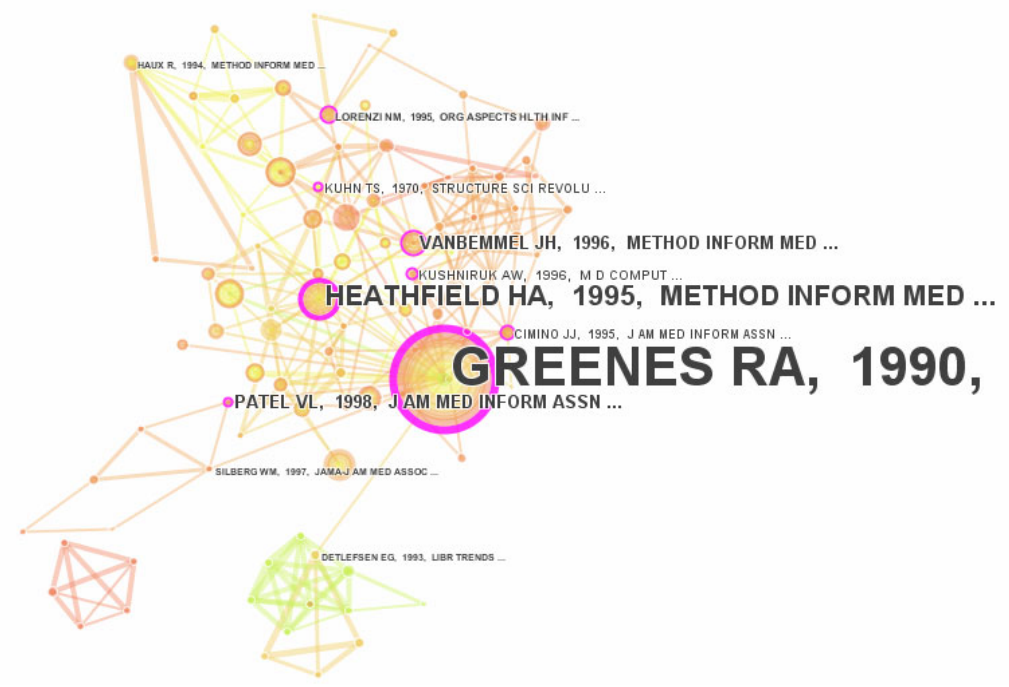

Fig. 3. Co-citation network of publications for 1970-2000 (5-year slice, parameters c, cc, ccv: $3,3,25$ ) (output from CiteSpace II)

A different pattern emerges in Figure 4, which shows a co-citation network of publications for 2001-2005. No longer is Greenes' 1996 article the most dominant; instead in terms of co-citations made to the article. It appears that Hunt's article [49] and Eysenbach's article [23] are the most co-cited in this period. It is interesting to note that, whereas for the 1970-2000 period the most important articles were theoretically-based, for the 2001-2005 period there seems to be a switch on emphasis to empirical and evidence-based research, as exemplified by Hunt's article. This observation is corroborated by a closer examination at other highly co-cited articles.

The rapid growth in publications and the switch from a theoretical to an empirical focus suggests that health informatics, as a discipline, has grown and developed in multiple ways, leading to a divergence in research themes and priorities. In addition, from Figure 4, it is possible to detect some clusters of publications. The group of publications connected by yellow links (top-left corner), whose co-citations began in 2004, seem much influenced by articles by Haux and Knaup [22] and Kulikowski [33] (see black highlighted oval). The Haux and Knaup article provided recommendations on health informatics education; whereas, Kulikowski has described some challenges of health informatics, at both micro and macro levels and from multiple perspectives. Both articles are the linkage articles between the yellow cluster and light blue cluster. It is interesting to note that the two most influential 
articles by Hunt and Eysenbach have direct connections with the orange, green, blue, and light blue clusters, but not with the yellow cluster.

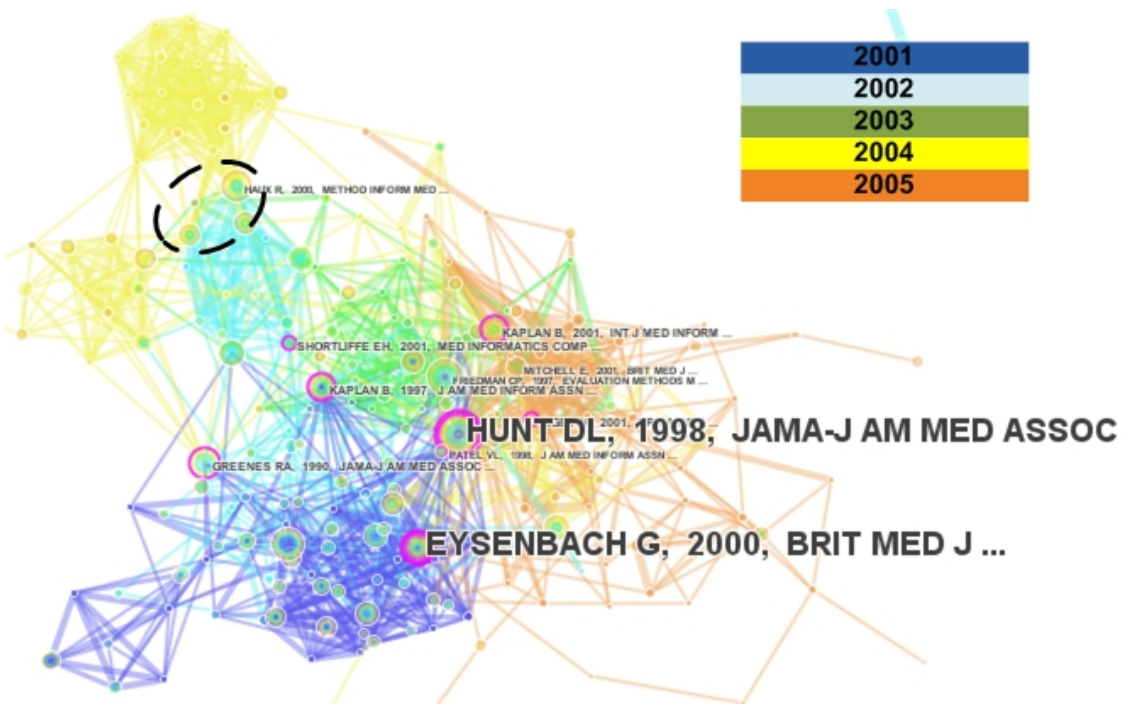

Fig. 4. Co-citation network of publications for 2001-2005 (1-year slice, parameters c, cc, ccv: 3, 3, 25) (output from CiteSpace II)

Similar patterns can be observed from the 2006-2009 period, where a greater number of articles seem to be empirically orientated-an observation confirmed by a rapid survey of the articles first co-cited during this period. The co-citation network is more complex than the one for 2001-2005 because of the large number of publications (see Table 1) and of co-citations. It is difficult to locate patterns within such complex visualization.

\section{Summary, Limitations, and Future Work}

In this paper, we have used two bibliometric tools to provide an overview of the research field of health informatics. A broad literature search was conducted on Web of Science database, producing a large number of results. The results were analyzed using the bibliometric tools HistCite and CiteSpace II. The analysis lead to the identification of patterns of growth, some of the most influential articles, the most prolific authors and research institutions, the most important publication venues, and some recent patterns of emphasis.

One limitation of the approach taken is that it excludes articles not found on the Web of Science database. In addition, the approach relies on the output from two bibliometric tools, both of which depend on citation or co-citation counts. Consequently, it does not take into account publications with low citation and cocitation counts, a common property with recent publications. A further limitation is 
that the approach can only hint at the reasons for the citation patterns because it does not examine in detail the content of the articles. Content analysis is required to infer reasons for citation patterns. Some reasons for citation are profound, but other reasons may be trivial because papers often are cited in a perfunctory fashion and are not essential to the goals of the citing paper. In addition, the output from CiteSpace II can be quite complex, and further exploration is required to determine specific patterns and trends in the body of literature.

An interesting avenue for future work would be to conduct a more detailed content analysis to discern how the important publications have shaped or still are shaping the field. Furthermore, a more detailed analysis could be conducted with CiteSpace II to locate emerging "research fronts" and "intellectual bases" in medical informatics research-where a research front is the state-of-the-art of a research area and an intellectual base is the publications cited by the research front. This analysis would allow us to understand the future evolution and research trends in health informatics.

\section{Acknowledgments}

The author gratefully acknowledges the valuable input and assistance from members of the National Information and Communication Technology Australia (NICTA) Cognitive and Organisational Systems Engineering (COSE) project. NICTA is funded by the Australian Government as represented by the Department of Broadband, Communications and the Digital Economy and the Australian Research Council through the ICT Centre of Excellence program.

\section{References}

1. Protti, D.: The synergism of health/medical informatics revisited. Methods of Information in Medicine (34), 441-445 (1995)

2. Deloitte: Queesnland Health: eHeatlh Strategy (2006)

3. Coiera, E.: Guide to Health Informatics, 2nd edn. Hodder Headline Group, London (2003)

4. Shortliffe, E.H., Cimino, J.J. (eds.): Biomedical Informatics: Computer Applications in Health Care and Biomedicine, 3rd edn. Springer, Heidelberg (2006)

5. De Bellis, N.: Bibliometrics and citation analysis: From the science citation index to cybermetrics. The Scarecrow Press, Inc., Lanham (2009)

6. Van Leeuwen, T.N., Visser, M.S., Moed, H.F., Nederhof, T.J., Van Raan, A.F.J.: The Holy Grail of science policy: Exploring and combining bibliometric tools in search of scientific excellence. Scientometrics 57(2), 257-280 (2003)

7. Bordons, M., Morillo, F., Gómez, I.: Analysis of cross-disciplinary research through bibliometric tools. In: Moed, H., Glänzel, W., Schmoch, U. (eds.) Handbook of Quantitative Science and Technology Research: The Use of Publication and Patent Statistics in Studies of S\&T Systems, pp. 437-456. Kluwer Academic Publishers, New York (2004)

8. Nederhof, A.J., Meijer, R.F.: Development of bibliometric indicators for utility of research to users in society: Measurement of external knowledge transfer via publications in trade journals. Scientometrics 32(1), 37-48 (1995)

9. Higher Education Funding Council for England. HEFCE: Research: REF: Bibliometrics (2010) 
10. Persson, O.: BIBEXCEL, Umeå, Sweden. BIBEXCEL A tool-box developed by Olle Persson (2010)

11. Chen, C.: CiteSpace II: Detecting and visualizing emergent trends and transient patterns in scientific literature. Journal of the American Society for Information Science and Technology 57(3), 359-377 (2006)

12. Garfield, E.: Historiographic mapping of knowledge domains literature. Journal of Information Science 30(2), 119-145 (2004)

13. Schildt, H.A., Mattsson, J.T.: A dense network sub-grouping algorithm for co-citation analysis and its implementation in the software tool Sitkis. Scientometrics 67(1), 143-163 (2006)

14. Chen, C.: CiteSpace: Visualizing patterns and trends in scientific literature (2010)

15. Garfield, E., Pudovkin, A.I., Istomin, V.I.: Mapping the Output of Topical Searches in the Web of Knowledge and the case of Watson-Crick. Information Technology and Libraries 22(4), 183-187 (2003)

16. HistCite Software LLC. HistCite, Bala Cynwyd, PA (2010)

17. Chen, C.: Information visualization: Beyond the horizon, 2nd edn. Springer, London (2006)

18. Reuters, T.: Web of Science - Science - Thomas Reuters (2010)

19. Dem, T.: MEDINFO 1974: First World Conference on Medical Informatics. Biomedical Engineering 9(11), 524-525 (1974)

20. Greenes, R.A., Shortliffe, E.H.: Medical informatics: an emerging academic discipline and institutional priority. Journal of the American Medical Association 263(8), 1114-1120 (1990)

21. Collen, M.F.: Origins of medical informatics. Western Journal of Medicine 145(6), 778-785 (1986)

22. Haux, R., Knaup, P.: Recommendations of the International Medical Informatics Association on education in health and medical informatics. Methods of Information in Medicine 39(3), 267-277 (2000)

23. Eysenbach, G.: Recent advances: Consumer health informatics. British Medical Journal 320(7251), 1713-1716 (2000)

24. Kaplan, B.: Evaluating informatics applications-some alternative approaches: theory, social interactionism, and call for methodological pluralism. International Journal of Medical Informatics 64(1), 39-55 (2001)

25. Gustafson, D.H., Hawkins, R., Boberg, E., Pingree, S., Serlin, R.E., Graziano, F., Chan, C.L.: Impact of a Patient-Centered, Computer-Based Health Information/Support System. American Journal of Preventive Medicine 16(1), 1-9 (1999)

26. Ammenwerth, E., Brender, J., Nykanen, P., Prokosch, H.U., Rigby, M., Talmon, J.: Visions and strategies to improve evaluation of health information systems - Reflections and lessons based on the HIS-EVAL workshop in Innsbruck. International Journal of Medical Informatics 73(6), 479-491 (2004)

27. van Bemmel, J.H.: Medical informatics, art or science? Methods of Information in Medicine 35(3), 157-172 (1996)

28. Kaplan, B.: Addressing organizational issues into the evaluation of medical systems. Journal of the American Medical Association 4(2), 94-101 (1997)

29. Martin-Sanchez, F., Iakovidis, I., Noraer, S., et al.: Synergy between medical informatics and facilitating genomic medicine for future bioinformatics: health care. Journal of Biomedical Informatics 37(1), 30-42 (2004)

30. Sittig, D.F.: Grand challenges in medical informatics. Journal of the American Medical Association 1(5), 412-413 (1994) 
31. Haux, R.: Aims and tasks of medical informatics. International Journal of Medical Informatics 44(1), 9-20 (1997)

32. Lorenzi, N.M., Riley, R.T., Blyth, A.J.C., Southon, G., Dixon, B.J.: Antecedents of the people and organizational aspects of medical informatics: Review of the literature. Journal of the American Medical Association 4(2), 79-93 (1997)

33. Kulikowski, C.A.: The micro-macro spectrum of medical informatics challenges: From molecular medicine to transforming health care in a globalizing society. Methods of Information in Medicine 41(1), 20-24 (2002)

34. Stead, W.W., Haynes, R.B., Fuller, S., et al.: Designing medical informatics research and library-resource projects to increase what is learned. Journal of the American Medical Association 1(1), 28-33 (1994)

35. Heathfield, H.A., Wyatt, J.: The road to professionalism in medical informatics: A proposal for debate Methods of Information in Medicine 34(5), 426-433 (1995)

36. van Bemmel, J.H.: The structure of medical informatics. Medical Informatics 9(3-4), 175-180 (1984)

37. Reichertz, P.L.: Preparing for change: Concepts and education in medical informatics. Computer Methods and Programs in Biomedicine 25(2), 89-101 (1987)

38. Haux, R., Dudeck, J., Gaus, W., et al.: Recommendations of the German association for medical informatics, biometry and epidemiology for education and training in medical informatics. Methods of Information in Medicine 31(1) (1992)

39. Heathfield, H.A., Wyatt, J.: Philosophies for the design and development of clinical decision-support systems. Methods of Information in Medicine 32(1), 1-8 (1993)

40. Friedman, C.P.: Where's the science in medical informatics? Journal of the American Medical Association 2(1), 65-67 (1995)

41. Hasman, A., Haux, R., Albert, A.: A systematic view on medical informatics. Computer Methods and Programs in Biomedicine 51(3), 131-139 (1996)

42. McDonald, C.J.: The barriers to electronic medical record systems and how to overcome them. Journal of the American Medical Informatics Association 4(3), 213-221 (1997)

43. Cimino, J.J.: Desiderata for controlled medical vocabularies in the twenty-first century. Methods of Information in Medicine 37(4-5), 394-403 (1998)

44. Ball, M.J., Lillis, J.: E-health: transforming the physician/patient relationship. International Journal of Medical Informatics 61(1), 1-10 (2001)

45. Patel, V., Arocha, J., Kaufman, D.: Review? A primer on aspects of cognition for medical informatics. Journal of the American Medical Informatics Association 8(4), 324-343 (2001)

46. Maojo, V., Kulikowski, C.: Bioinformatics and medical informatics: Collaborations on the road to genomic medicine? Journal of the American Medical Informatics Association 10(6), 515-522 (2003)

47. Ammenwerth, E., de Keizer, N.: An inventory of evaluation studies of information technology in health care - Trends in evaluation research 1982-2002. Methods of Information in Medicine 44(1), 44-56 (2005)

48. Haux, R.: Health information systems - past, present, future. International Journal of Medical Informatics 75(3-4), 268-281 (2006)

49. Hunt, D.L., Haynes, R.B., Hanna, S.E., Smith, K.: Effects of Computer-Based Clinical Decision Support Systems on Physician Performance and Patient Outcomes: A Systematic Review. Journal of the American Medical Association 280(15), 1339-1346 (1998) 